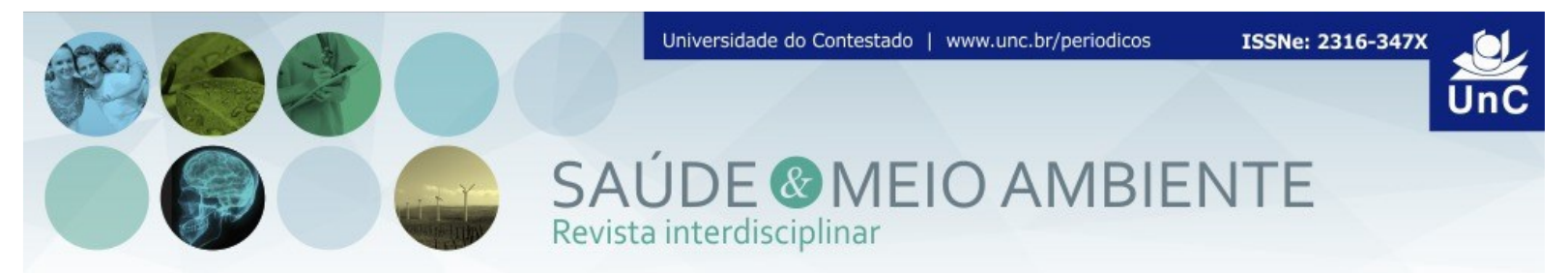

\title{
EDUCAÇÃO ALIMENTAR E PERFIL NUTRICIONAL DE ACADÊMICOS DE NUTRIÇÃO DA UNIVERSIDADE FEDERAL DO AMAZONAS
}

\section{FOOD EDUCATION AND NUTRITIONAL STATUS OF NUTRITION ACADEMIC AT THE AMAZONAS FEDERAL UNIVERSITY}

\author{
Katiane Melo dos Santos 1 \\ Karoline Santana de Freitas ${ }^{2}$ \\ Klenicy Kazumy de Lima Yamaguchi ${ }^{3}$
}

\begin{abstract}
RESUMO
O ingresso de alunos na faculdade dificulta a conciliação entre os estudos e uma boa alimentação, de forma que o tempo para se alimentar fica cada vez menor. O objetivo deste estudo foi identificar o estado nutricional dos acadêmicos de nutrição no Instituto de Saúde e Biotecnologia da Universidade Federal do amazonas (ISB/UFAM), através de cálculos de índice de massa corpórea (IMC), relação cintura quadril (RCQ), parâmetro relação cintura estatura (RCE), Questionário do Recordatório 24 hora e Questionário de frequência alimentar (QFA) caracterizando-se um estudo transversal, de cunho descritivo com uma abordagem quantitativa e qualitativa. Participaram da amostra 60 alunos selecionados aleatoriamente, com grande incidência de alunos eutróficos. Em relação circunferência da Cintura identificando 18,3\% da população estudada tem risco para doenças cardiovasculares e obesidade. Dentre os inquéritos a Relação Cintura - Quadril (RCQ), constatou apenas 13,3\% da amostra com risco e a relação cintura-estatura foi encontrado apenas $13,3 \%$ da amostra total para doenças cardiovasculares e obesidade. Já em relação ao QFA foi encontrado baixa ingestão de alguns grupos de alimentos e no Recordatório 24 horas foram encontradas baixa ingestão de macronutrientes. Sendo assim, a alimentação dos acadêmicos de nutrição analisados foi considerada inadequada. Os resultados encontrados sugerem que o conhecimento nutricional obtidos no decorrer do curso, não é capaz de influenciar o comportamento alimentar desses acadêmicos.
\end{abstract}

Palavras-chave: Estado nutricional. Acadêmicos de nutrição. Avaliação antropométrica

\footnotetext{
1Bacharela em Nutrição, Instituto de Saúde e Biotecnologia - ISB/UFAM. Universidade Federal do Amazonas. Coari. Amazonas. Brasil. Orcid: https://orcid.org/0000-0001-6711-0392. E-mail: katty.ufam@gmail.com

2 Graduada e Escpecialista em Nutrição. Professora Especialista Substituta do Instituto de Saúde e Biotecnologia. Universidade Federal do Amazonas. Coari, Amazonas. Brasil. OPRCID: https://orcid.org/0000-0003-3548-8045. E-mail: karol freitas 84@hotmail.com

3 Professora Doutora do Instituto de Saúde e Biotecnologia Universidade Federal do Amazonas. Coari, Amazonas. Brasil. E-mail: klenicy@gmail.com
} 


\begin{abstract}
The admission of students to college makes it difficult to reconcile studies and a good diet, so that the time to eat is getting shorter and shorter. The aim of this study was to identify the nutritional status of nutrition students at the Institute of Health and Biotechnology of the Federal University of amazonas (ISB / UFAM), through calculations of body mass index (BMI), waist-hip ratio (WHR), parameter waist-toheight ratio $(R C E)$, 24-hour recall questionnaire and food frequency questionnaire (FFQ) featuring a cross-sectional, descriptive study with a quantitative and qualitative approach. Sixty randomly selected students participated in the sample, with a high incidence of eutrophic students. Regarding waist circumference, identifying $18.3 \%$ of the studied population is at risk for cardiovascular diseases and obesity. Among the surveys on the Waist - Hip Ratio (WHR), it was found that only $13.3 \%$ of the sample was at risk and the waist - height ratio was found only $13.3 \%$ of the total sample for cardiovascular diseases and obesity. Regarding the FFQ, low intake of some food groups was found and in the 24-hour recall, low intake of macronutrients was found. Thus, the nutrition students' diet analyzed was considered inadequate. The results found suggest that the nutritional knowledge obtained during the course is not able to influence the eating behavior of these students.
\end{abstract}

Keywords: Nutritional status. Nutrition academics. Anthropometric assessme.

\title{
1 INTRODUÇÃO
}

Com a inserção na Universidade e a saída da casa dos pais, estudos descrevem que os jovens costumam sentir dificuldades em prover sua própria alimentação, pois são influenciados por diversos fatores como novas relações sociais, desempenho acadêmico, estresse, instabilidade psicossocial, modismos dietéticos, omissão de refeições, consumo de fast foods, consumo de álcool e cigarros, com isso postergando a importância de uma alimentação saudável[1]. O estilo de vida universitário e o padrão alimentar estão associados ao adverto de Doenças Cronicas não Transmissivel (DCNT). ${ }^{2]}$

O estado nutricional de um indivíduo é expresso pelo grau em que as necessidades fisiológicas nutricionais são alcançadas, para que deste modo ocorra a conservação da composição corporal e o funcionamento adequado de órgãos e sistemas, para que resulte no equilíbrio entre a necessidade, ingestão e utilização de nutrientes ${ }^{[3]}$.

De acordo com a OMS, os indicadores antropométricos devem ser utilizados na determinação do estado nutricional e de saúde de indivíduos e coletividades, sendo importantes no diagnóstico e acompanhamento da situação nutricional e crescimento corporal[4].

O nutricionista é capacitado para atuar como educador, assumindo um importante papel de auxiliar os indivíduos a estabelecer práticas de hábitos e consumo 
alimentares adequados às suas necessidades nutricionais, recursos alimentares locais e o padrão cultural de cada indivíduo[5]. Por ser um profissional frequentemente associado à imagem de um corpo saudável e magro, principalmente por estar vinculado ao conhecimento da alimentação, nutrição e saúde, o nutricionista possui uma grande cobrança em modificar a ingestão alimentar para atingir um padrão preestabelecido $^{[1]}$

O conhecimento sobre o tema tende a proporcionar uma alimentação mais balanceada com ingestão de determinados grupos de alimentos essenciais para o organismo. Dessa forma, espera-se que os acadêmicos iniciantes ou concludentes do curso de nutrição, sendo os futuros profissionais da área da saúde e formadores de conceitos e práticas, apresentem uma dieta mais equilibrada, além de serem atuantes na comunicação de saberes sobre hábitos de vida saudável para a população no geral. Apesar dos conhecimentos adquiridos durante a graduação, ainda é possível verificar hábitos alimentares inadequados devido a conciliação de tempo com as disciplinas da universidade ${ }^{[6]}$

Assim, faz-se necessário estudos do estado nutricional voltados para essa temática em relação aos acadêmicos de nutrição. Diante do exposto, o presente estudo teve como objetivo a identificação do perfil nutricional e hábitos alimentares dos acadêmicos de nutrição da Universidade Federal do Amazonas.

\section{MATERIAL E MÉTODOS}

Trata-se de um estudo transversal de cunho descritivo com uma abordagem quantitativa e qualitativa, envolvendo os acadêmicos do curso de nutrição do Instituto de Saúde e Biotecnologia da Universidade Federal do Amazonas (ISB-UFAM) no município de Coari - Amazonas. A População alvo do estudo foi composta por acadêmicos do $1^{\circ}, 3^{\circ}, 5^{\circ}, 7^{\circ}$, e $9^{\circ}$ período do respectivo curso, no segundo semestre de 2017. Para o estudo participaram os estudantes de ambos os sexos, maior ou igual a 18 até 32 anos, que voluntariamente aceitaram participar do mesmo. A relevância desse estudo se deu a partir da ausência de estudos sobre perfis nutricionais no município de Coari em relação aos acadêmicos de nutrição do ISB - UFAM. Tais informações são importantes para comunidade acadêmica e para o próprio município de Coari, levando em consideração esse aumento de jovens na comunidade acadêmica, incentivando para trabalhos futuros e pesquisas relacionados a perfil nutricional e orientações para estudantes universitários, ajudando na diminuição dos indicativos encontrados.

Os dados foram coletados por meio de uma ficha de avaliação de Perfil Nutricional, Questionário de frequência alimentar (QFA) e Registro alimentar 24 horas, aplicado individualmente.

Foi utilizado o Questionário de Frequência Alimentar (QFA) validado em um estudo com adultos. Composto por 180 itens, referem-se à frequência do consumo de frutas, verduras, legumes, leguminosas, carnes (boi, frango, peixe e ovos), leite e seus 
derivados, doces e embutidos. A avaliação do consumo dos alimentos foi comparada com outros estudos já realizados. A frequência de consumo é caracterizada por dia, semana mês ou nunca, Slater et al. ${ }^{[7]}$. A ficha de Avaliação do perfil nutricional foi utilizada para a coleta de dados pessoais, em que consta a idade, sexo, período, peso, altura, circunferência da cintura e circunferência do quadril.

O registro alimentar 24 horas possui informações básicas como horário das refeições e locais das refeições realizadas. Neste registro coletou-se dados de todos os alimentos consumidos no período de 24 horas prévias, desde a primeira até a última refeição realizada neste intervalo de tempo (café da manhã, lanche da manhã, almoço, lanche da tarde, jantar e ceia).

Os alimentos relatados pelos entrevistados foram registrados em medidas caseiras ou gramas e o registro alimentar obtido nunca foi de períodos atípicos dos acadêmicos.

Para aferir o peso e altura utilizou-se balança modelo digital portátil, com capacidade para $180 \mathrm{~kg}$, precisão de 100 gramas da marca Sanny. Os alunos foram pesados descalços e usando roupas leves. A estatura foi aferida com estadiometro portátil da marca sanny com os alunos descalços, a cabeça posicionada na posição de Frankfurt, admitindo variação máxima de $0,5 \mathrm{~cm}$. A aferição da cintura e quadril foi realizada com fita métrica portátil da marca sanny com variação máxima de $0,5 \mathrm{~cm}$.

Para avaliar a adequação nutricional todos os alimentos contidos no registro alimentar 24 horas foram modificados para gramas e calculadas as porcentagens. Para o consumo adequado foi definida a ingestão de 50 a $60 \%$ de carboidratos, 10 a $15 \%$ de proteínas e de $25-30 \%$ de lipídeos do total de calorias proposto pelo Guia alimentar da População Brasileira.

Para os cálculos destas porcentagens foi utilizado o programa DietBox que é um sistema nutricional, utilizado para cadastramento e cálculo de dietas, o qual contêm uma lista de alimentos já cadastrados. Tendo como referência a Tabela Brasileira de composição de alimentos (TACO).

Após a verificação das medidas antropométricas, a Avaliação do estado nutricional foi calculado a partir do Índice de Massa Corporal (IMC), o qual baseia-se na relação peso corporal $(\mathrm{kg})$ /estatura $(\mathrm{m} 2)$, adotando como critério de classificação proposta pela Organização Mundial de Saúde (OMS). Para obtenção de resultado de Relação Cintura Quadril (RCQ), circunferência da Cintura foi medido o perímetro da cintura que foi medido na circunferência menor existente entre a última costela e a crista ilíaca, e do quadril na maior região do glúteo, com auxílio de uma fita métrica e seus resultados foram comparados com resultados padrões de tabelas referência também da organização Mundial da saúde (OMS).

Para relevâncias dos dados foi utilizado outro índice antropométrico de obesidade e relação com doenças cardiovasculares (Relação Cintura - Estatura (RCEst), sendo ele comparado com outros índices antropométricos já citados nesse trabalho. A RCEst foi calculada com os dados já disponibilizados pelos alunos na ficha de avaliação do perfil Nutricional. A RCEst é calculada por uma simples divisão de cintura por estatura. O interesse para o seu uso nesse projeto está no pressuposto de 
que, para uma dada estatura, á uma quantidade aceitável de gordura na região do tronco. Sendo esse índice antropométrico pouco estudado no Brasil, a tabela de referência usada foi do artigo - Index of central obesity - A novel parameter, comparando também com o estudo realizado no Brasil. O perfil nutricional padrão foi elaborado baseado na média dos valores nutricionais adquiridos nas pesquisas.

O referido projeto foi submetido e aprovado pelo Comitê de Ética em Pesquisa da Universidade Federal do Amazonas - UFAM, sob o parecer $n^{\circ} 2.311 .769$. sob o critério de inclusão Participarão do estudo, os acadêmicos do curso de nutrição que concordarem e assinarem o Termo de Consentimento Livre e Esclarecido, que sejam maiores de 18 anos, de ambos os sexo, que estejam regulamente matriculados no curso de Nutrição no Instituto de Saúde e Biotecnologia ISB- UFAM, sendo em vista que fará uma participação voluntária e tendo conhecimento de seus riscos e benefícios. Não participarão do estudo aqueles alunos que apesar de preencherem alguns dos critérios de inclusão, apresentam características que podem interferir na coleta de dados tais como discordarem e não assinarem o Termo de Consentimento Livre e Esclarecido, que sejam menores de 18 anos e que não estejam matriculados no Curso de Nutrição no Instituto de Saúde e Biotecnologia - ISB - UFAM. Desta forma não puderam participar desta pesquisa (de acordo com a Res.466/2012). Todas os estudantes confirmaram sua participação na pesquisa mediante assinatura do termo de consentimento livre e esclarecido (TCLE).

\section{RESULTADOS}

A amostra foi constituída por 60 acadêmicos do Curso de bacharel em Nutrição com a seguinte faixa etária: 18 anos (23,3\%), 19 a 23 anos (55\%), 24 a 29 anos $(18,3 \%)$ e 32 anos (3,4\%). Sendo composto por $44(73,3 \%)$ do sexo Feminino e 16 $(26,7 \%)$ do sexo masculino, sendo a porcentagem do sexo feminino estudada bem superior ao do sexo masculino. A tabela 1 apresenta a caracterização geral da amostra desta pesquisa. 
Tabela 1 - Caracterização Geral da população

\begin{tabular}{|c|c|c|c|}
\hline Questão & Opções & Quantidade & $\%$ \\
\hline \multirow[t]{5}{*}{ 1. Periodização } & $1^{\circ}$ & 19 & 31,7 \\
\hline & $3^{\circ}$ & 08 & 13,3 \\
\hline & $5^{\circ}$ & 16 & 26,7 \\
\hline & $7^{\circ}$ & 10 & 16,7 \\
\hline & $9^{\circ}$ & 07 & 11,6 \\
\hline \multirow[t]{3}{*}{ 2. Local da Refeição } & Restaurante Universitário (R.U) & 15 & 25,0 \\
\hline & Restaurante Universitário (R.U) + Casa & 17 & 28,3 \\
\hline & Apenas Casa & 28 & 46,7 \\
\hline \multirow{4}{*}{$\begin{array}{l}\text { 3. Quantidade de } \\
\text { Refeição /Dia }\end{array}$} & 3 refeições & 12 & 20,0 \\
\hline & 4 refeições & 22 & 36,7 \\
\hline & 5 refeições & 16 & 26,7 \\
\hline & 6 refeições & 10 & 16,6 \\
\hline \multirow{6}{*}{$\begin{array}{l}\text { 4. Distribuição/ } \\
\text { Refeição }\end{array}$} & Desjejum & 55 & 91,7 \\
\hline & Lanche da Manhã & 12 & 20,0 \\
\hline & Almoço & 60 & 100 \\
\hline & Lanche da tarde & 49 & 81,7 \\
\hline & Jantar & 56 & 93,3 \\
\hline & Ceia & 23 & 38,3 \\
\hline \multirow[t]{12}{*}{ 5. horário das refeições } & Desjejum (7:00 às 8:30h) & 39 & 70,9 \\
\hline & Desjejum (a partir de 8:30h) & 16 & 29,1 \\
\hline & Lanche da Manhã (9:30 às 10:30h) & 10 & 83,3 \\
\hline & Lanche da Manhã (a partir de 10:30h) & 2 & 16,7 \\
\hline & Almoço (11:00 às 13:30h) & 58 & 96,7 \\
\hline & Almoço (a partir de 13:30h) & 2 & 3,3 \\
\hline & Lanche da tarde (15:00 às 16:30h) & 37 & 75,5 \\
\hline & Lanche da tarde (a partir de 16:30h) & 12 & 24,5 \\
\hline & Jantar (17:30 às 20:30h) & 36 & 64,3 \\
\hline & Jantar (a partir de 20:30h) & 20 & 35,7 \\
\hline & Ceia (21:00 às 22:00h) & 11 & 47,8 \\
\hline & Ceia (a partir de 22:00h) & 12 & 52,2 \\
\hline
\end{tabular}

Fonte: Dados da pesquisa

O percentual de acadêmicos pelo período cursado possibilitou uma melhor observação entre os resultados, sendo o $1^{\circ}$ período com a maior população e o $9^{\circ}$ período a menor população estudada. Foram analisados o local e quantidade de refeições realizadas pelos acadêmicos. Em relação ao local de refeição (item 2), podese notar que a maioria dos acadêmicos estudados fazem as refeições tanto no restaurante universitário quanto em casa, ressaltando que $25 \%$ desses acadêmicos dependem inteiramente do restaurante universitário e os outros $75 \%$ alternam entre restaurante e suas residências.

Pode-se observar que a maioria dos acadêmicos realizam 04 refeições por dia, destas, incluem-se as refeições consideradas "mais importantes" (almoço e janta), e $43,3 \%$ dessa população, realiza a quantidade de refeição recomendada, entre 5 a 6 . 
$\mathrm{Na}$ avaliação da distribuição das refeições (item 4), 80\% dos acadêmicos não realizam o lanche da manhã pela disponibilização de horário, alegando estarem em aula nesse período. Apenas $91,7 \%$ dos acadêmicos realizam o desjejum. Todos $(100 \%)$ os indivíduos investigados realizam o almoço, $93,3 \%$ tem o habito de jantar e apenas $38,3 \%$ realizam a ceia ou lanche da noite.

Verificou-se que os acadêmicos avaliados apresentam rotina com horários de refeições distintas e diferentes dos horários pré-estabelecidos (tabela 2). Os alunos que dependem ou preferem se alimentar no restaurante universitário tendem a seguir os horários estabelecido pelo próprio restaurante como podemos analisar no item 5 (tabela 1).

Ao analisar a quantidade de refeições por local de refeições realizadas (figura 1), pode-se verificar que os alunos preferem realizar o desjejum e o jantar em suas residências e o almoço e lanche da tarde na Universidade (restaurante universitário).

$\mathrm{Na}$ análise nutricional (tabela 2), a prevalência geral de eutróficos foi de $75 \%$ da amostrada estudada, sendo encontrada uma porcentagem significante de Sobrepeso (15\%), de obesidade grau I $(6,7 \%)$ e uma porcentagem pequena, mas preocupante de desnutrição grau I (3,3\%).

Figura 1 - Quantidade pelo local das refeições realizadas pelos alunos

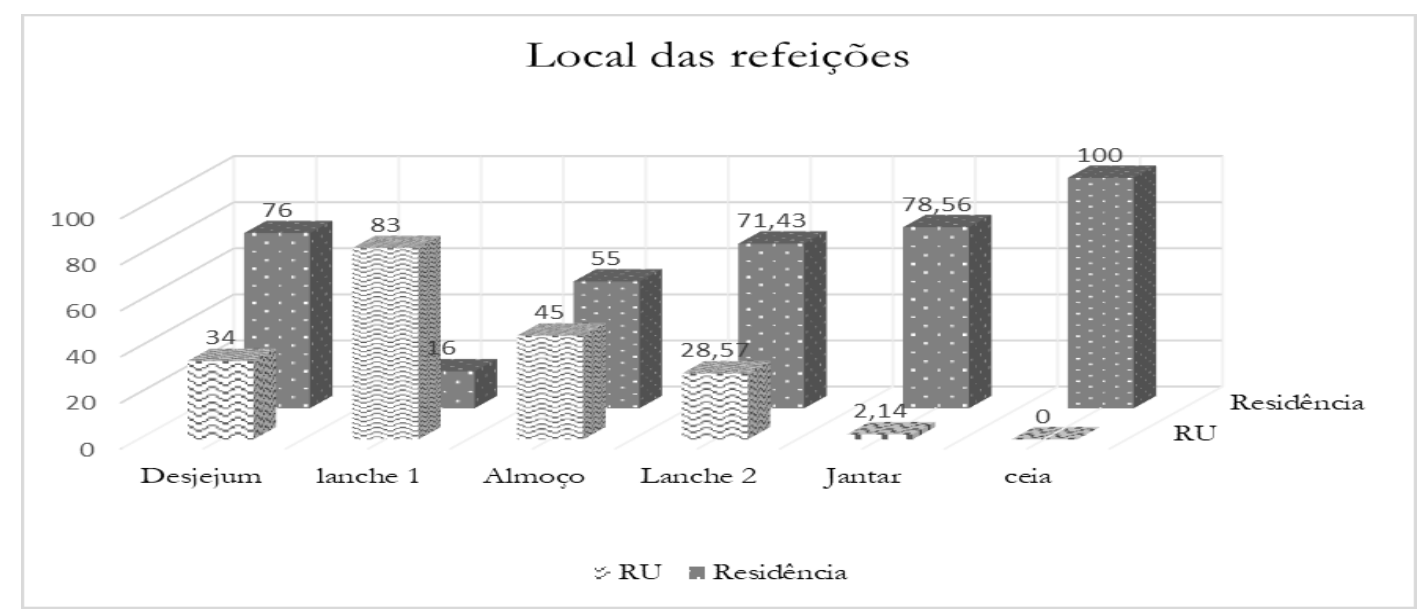

Fonte: Dados da pesquisa 
Tabela 2 - Estado Nutricional dos acadêmicos

\begin{tabular}{l|c|c|c}
\hline \multicolumn{1}{c|}{ Classificação } & Opções & Quantidade & $\%$ \\
\hline \multirow{4}{*}{ 1. Em relação ao IMC } & Desnutrição grau I & 02 & 3,3 \\
\cline { 2 - 4 } & Eutrofia & 45 & 75 \\
\cline { 2 - 4 } & Sobrepeso & 09 & 15 \\
\cline { 2 - 4 } & Obesidade grau I & 04 & 6,7 \\
\hline \multirow{2}{*}{\begin{tabular}{l} 
Circunferência da Cintura \\
\cline { 2 - 4 }
\end{tabular}} & Normal & 49 & 81,7 \\
\cline { 2 - 4 } & Elevada & 05 & 8,3 \\
\hline \multirow{2}{*}{ 3. Modo Geral } & Nuito elevada & 06 & 86,7 \\
\hline \multirow{2}{*}{$\begin{array}{l}\text { 4. Valores de calorias por } \\
\text { quantidade de alunos }\end{array}$} & Risco & 08 & 13,3 \\
\cline { 2 - 4 } & 1.000 a $1.500 \mathrm{kcal} / \mathrm{dia}$ & 04 & 41,7 \\
\cline { 2 - 4 } & 1.500 a $2.000 \mathrm{kcal} / \mathrm{dia}$ & 25 & 25 \\
\cline { 2 - 4 } & $>2.000 \mathrm{kcal} / \mathrm{dia}$ & 15 & 26,6 \\
\hline
\end{tabular}

Fonte: Dados da pesquisa

Os resultados apresentados na tabela 2 foram evidenciados pela divisão dos períodos acadêmicos (tabela 3 ). Observou-se uma maior incidência de eutróficos, tanto no $1^{\circ}$ quanto no $5^{\circ}$ período. Em todos os períodos analisados foram encontrados acadêmicos com sobrepeso, destacando $03^{\circ}$ período com acadêmicos com obesidade grau I e o $1^{\circ}$ período com detecção de alunos com desnutrição grau I.

Outro inquérito de avaliação nutricional utilizado foi a circunferência da Cintura, identificando que $8,3 \%$ da população estudada apresenta risco elevado e $10 \%$ risco muito elevado para doenças cardiovasculares e obesidade. Vale ressaltar que a maioria dessa população, $81,7 \%$, estão em estado normal. Foi analisado por esse mesmo inquérito antropométrico, o perfil nutricional dos acadêmicos por período estudado, encontrado uma porcentagem significativa de cintura elevada no $7^{\circ}$ período e muito elevada no $1^{\text {a }}$ período (item 2). Verifica-se que estes dados apontam para um índice preocupante, pois os alunos do $7^{\circ}$ período têm um conhecimento sobre alimentação superior aos do $1^{\circ}$ período que acabaram de entrar na universidade.

Analisou-se esse mesmo inquérito antropométrico por gênero da amostra estudada, onde foi observada que entre mulheres esse índice é predominante, com risco elevado ou muito elevado da circunferência da cintura no sexo feminino $(16,67 \%)$ e apenas uma amostra foi detectada no sexo masculino $(1,67 \%)$, apontando que há um estado nutricional preocupante no sexo feminino. A média de circunferência de cintura pelos alunos foi de $77,50 \mathrm{~cm}$, sendo considerada sem riscos segundo a classificação da OMS. 
Tabela 3 - Estado Nutricional dos acadêmicos

\begin{tabular}{l|c|c|c|c|c|c}
\hline \multicolumn{1}{c|}{ Estado nutricional } & Opções & $\mathbf{1}^{\mathbf{0}}$ & $\mathbf{3}^{\mathbf{0}}$ & $\mathbf{5}^{\mathbf{0}}$ & $\mathbf{7}^{\mathbf{0}}$ & $\mathbf{9}^{\mathbf{0}}$ \\
\hline \multirow{3}{*}{ Em relação ao IMC } & Desnutrição grau I & 02 & - & - & - & - \\
\cline { 2 - 7 } & Eutofria & 13 & 07 & 13 & 07 & 05 \\
\cline { 2 - 7 } & Sobrepeso & 02 & 01 & 02 & 02 & 02 \\
\cline { 2 - 7 } & Obesidade grau I & 02 & - & 01 & 01 & - \\
\hline \multirow{2}{*}{$\begin{array}{l}\text { Em relação a } \\
\text { Circunferência da Cintura }\end{array}$} & Normal & 14 & 08 & 14 & 06 & 07 \\
\cline { 2 - 7 } & Elevada & 01 & - & - & 04 & - \\
\cline { 2 - 7 } & Muito elevada & 04 & 02 & - & - & - \\
\hline \multirow{2}{*}{$\begin{array}{l}\text { Relação entre Cintura - } \\
\text { Quadril (RCQ) }\end{array}$} & Normal & 15 & 07 & 15 & 08 & 07 \\
\cline { 2 - 7 } & Risco & 04 & 01 & 01 & 02 & - \\
\hline
\end{tabular}

Fonte: Dados da pesquisa

Avaliando a relação cintura - quadril, verificou-se que $13,3 \%$ dos acadêmicos apresentam riscos para doenças cardiovasculares e predisposição para obesidade e $86,7 \%$ destes alunos apresentam quadro sem risco para essas patologias.

Este indicador foi analisado também por período acadêmico, onde detectou-se 04 alunos com risco no $1^{\circ}$ período e no $3^{\circ}$ e $5^{\circ}$ período, apenas 01 indivíduo apresentou essa predisposição. Analisou-se por gênero e foi observado que na amostragem utilizada houve uma exclusividade no sexo feminino. A média de circunferência de cintura pelos alunos foi de $0,81 \mathrm{~cm}$ e a média de RCQ pelos alunos foi de $0,81 \mathrm{~cm}$, sendo considerada sem riscos segundo a classificação da OMS.

Um inquérito antropométrico de pouco estudo, mas relevante e associado a distribuição de gordura abdominal e risco de doenças cardiovasculares também utilizado nessa pesquisa foi a relação cintura-estatura (RCEst). Verificou-se que $13 \%$ da amostragem apresenta risco, sendo esse percentual composto totalmente por mulheres. Pode-se observar uma quantidade significante de acadêmicas apresentam risco deste índice. A média de circunferência de cintura pelos alunos foi de 0,47 , sendo considerada sem riscos segundo o ponto de corte utilizado neste estudo.

A avaliação do QFA, entre os Cereais, o mais consumido entre os acadêmicos foi o arroz comum (85\% diário) e o menos consumido foi o arroz integral $(18,3 \%$ mensal). No grupo das leguminosas o feijão foi a única leguminosa constituinte do hábito alimentar dos estudantes pesquisados. Entre os vegetais pesquisados os mais consumidos pelos acadêmicos diariamente foram: alho $(81,6 \%)$, cebola $(76,7 \%)$, cheiro verde $(50 \%)$, pimenta de cheiro $(40 \%)$, tomate $(53,4 \%)$, pepino $(25 \%)$, couven(16,7\%) e alface $(33,3 \%)$, sendo que esses alimentos foram os com maior percentual de consumo em relação as variáveis desse grupo. No grupo dos tubérculos esses alimentos são consumidos com menos frequências pelos acadêmicos diariamente, os mais consumidos semanalmente foram a macaxeira $(43,3 \%)$ e a batata inglesa $(41,7 \%)$. Entre o grupo das frutas a banana $(28,3 \%)$ e a manga $(18,3 \%)$ foram as únicas frutas consumidas com mais frequência e diariamente pelos acadêmicos. As outras frutas são consumidas, mas, semanalmente por esses estudantes. 
Dentre o grupo das oleaginosas a pouco consumo diariamente e semanalmente, os acadêmicos relatam o maior consumo por mês. No meio do grupo das Farinhas, féculas e massas os produtos mais consumidos são a farinha de mandioca $(51,7 \%)$ e o macarrão $(33,3 \%)$. No grupo de panificados o pão caseiro $(38,3 \%)$ e pão francês $(58,3 \%)$ são predominantes no consumo diário desses acadêmicos.

O consumo diário de ovos $(46,7 \%)$ entre os estudantes foi alto quando comparado com o consumo carnes bovinas $(21,7 \%)$, de peixes $(6,7 \%)$, e aves $(35 \%)$. O leite integral em pó está entre os laticínios mais consumidos pelos estudantes diariamente $(40 \%)$, sendo que todos consumem algum tipo de leite sempre. Os resultados encontrados no registro alimentar 24 horas (recordatório) podem ser visualizados na tabela 3 . Os valores de calorias indicados para este estudo foram baseados na pirâmide alimentar brasileira adaptada pela Sônia Tucunduva de 2000 kcal/dia. Para brasileiros saudáveis.

Tabela 4 - Questionário do recordatório 24 horas dos discentes.

\begin{tabular}{l|c|c|c|c|c}
\hline \multicolumn{1}{c|}{ Estado nutricional } & $\mathbf{1}^{\mathbf{0}}$ & $\mathbf{3}^{\mathbf{0}}$ & $\mathbf{5}^{\mathbf{0}}$ & $\mathbf{7}^{\mathbf{0}}$ & $\mathbf{9}^{\mathbf{0}}$ \\
\hline Calorias (kcal/dia) & 1.754 & 1.530 & 1.884 & 1.845 & 1.981 \\
\hline Carboidratos ingeridos (\%) & 48,06 & 38,11 & 38,21 & 42,29 & 49,75 \\
\hline Proteínas ingeridas (\%) & 13,2 & 11,83 & 16,46 & 12,9 & 17,3 \\
\hline Lipídeos ingeridos (\%) & 24,33 & 28,71 & 31,3 & 33,25 & 31,40 \\
\hline Fibras (g/dia) & 12,45 & 14,35 & 10,53 & 15,45 & 20,62 \\
\hline
\end{tabular}

Fonte: Dados da pesquisa

A média de calorias ingeridas pelos alunos no modo geral, foi de $1.721,41$ $\mathrm{kcal} /$ dia. Dentre os valores obtidos, a quantidade de calorias mais consumidas variaram de 1.000 a $1.500 \mathrm{kcal}$ dia, sendo consideras abaixo da quantidade recomendada.

O consumo de carboidratos definido como o consumo ideal variou entre 50 a $60 \%$ do total de calorias diárias. A média de Carboidratos ingeridos pelos alunos foi de $43,26 \%$. Esses resultados demonstram uma grande prevalência $(68,3 \%)$ de acadêmicos que não ingerem a quantidade de carboidratos adequada, onde 18,4\% consomem acima do recomendando, principalmente nos alunos do $9^{a}$ período, e apenas $13,3 \%$ afirmaram utilizar a quantidade preconizada.

$\mathrm{Na}$ análise de proteínas, a média de calorias ingeridas pelos alunos foi de $14,38 \%$, variando conforme o período. Esse micronutriente foi o único ingerido de forma adequada segundo as recomendações diárias, com prevalência média no valor de $14 \%$.

A média de Lipídeos ingeridas pelos alunos foi de $29,09 \%$, estando dentro da margem do percentual recomendado ( 25 a $30 \%$ das calorias diárias). O consumo alto de lipídeos ( $>30 \%$ do consumo total de calorias) foi de $31,7 \%$, e 48,3 estão abaixo desse consumo diário de lipídeos, sendo apenas $20 \%$ dos alunos que consomem os lipídeos de forma recomendada. A média de lipídeos considerada alta, deu-se por 
causa que nesses $31,7 \%$ de alunos que consomem acima da recomendação, ultrapassando mais de $50 \%$ do recomendado.

Notou-se que a quantidade de fibras ingeridas pelo acadêmico é de baixo consumo, sendo recomendados o total de 20 a 30 gramas de fibras por dia. A média de fibras ingeridas pelos alunos no modo geral de 13,64 g/dia.

O consumo de fibras inadequado ressaltou em $78,3 \%$ da amostra estudada que consomem < 20 a 30 gramas de fibras por dia, sendo que apenas o $9^{\circ}$ período chega mais próximo do consumo adequado de fibras. Para elaboração do perfil nutricional dos acadêmicos de nutrição, utilizou-se a média dos valores encontrados nas pesquisas.

\section{DISCUSSÃO}

O consumo alimentar fora de casa está associado a hábitos alimentares menos saudáveis e a desequilíbrios nutricionais. Estudos indicam que as refeições realizadas fora de casa são desiquilibradas, pois incluem grandes quantidades de alimentos, são mais calóricas, possuem altos níveis de gordura total e saturada e contem baixos níveis de fibras, cálcio e ferro, referente ao baixo consumo de leite, frutas e hortaliças ${ }^{[8]}$.

Houve uma grande prevalência de mulheres nesta pesquisa $(73,3 \%)$, sendo que $90 \%$ do curso de nutrição são mulheres. De acordo com Feitosa et al. ${ }^{[9]}$, há um predomínio quase absoluto das mulheres na área da saúde, principalmente no curso de Nutrição. Esse predomínio pode ser explicado pelo fato de que as mulheres apresentam maior cuidado com a saúde quando comparadas aos homens.

Em relação às medidas antropométricas, de acordo com o IMC, a maioria dos acadêmicos analisados apresentou valores adequados (75\%), sendo apenas encontrados 3,3\% de Desnutrição grau I, $15 \%$ de sobrepeso e $6,7 \%$ de Obesidade grau I. Ramos ${ }^{[10]}$, num estudo realizado com universitários, obteve resultados semelhantes a este estudo em relação ao IMC. Na pesquisa, verificou-se que $77,1 \%$ dos estudantes apresentavam-se eutróficos, $15 \%$ com baixo peso, 7,9 com sobrepeso/obesidade.

Munhoz et al. ${ }^{[11]}$, descrevem em seu estudo que $74 \%(n=50)$ apresentavam normalidade, seguido de $13 \%(n=9)$ de excesso de peso, $7 \%(n=5)$ desnutrição e $6 \%$ $(n=4)$ de obesidade. Dados semelhantes aos encontrados por Gasparetto \& Silva[5], com $75 \%$ de eutrofia, $10 \%$ sobrepeso e desnutrição e $5 \%$ obesidade, e por Souza [12], com $79,3 \%$ eutrofia, $12,1 \%$ de sobrepeso/obesidade e $8,6 \%$ de baixo peso. Ambos apresentando predominância de eutrofia, assim como observada no atual estudo.

Feitosa et al. e Simão et al., ${ }^{[9,13]}$, confirmam os resultados encontrado em estudos envolvendo universitários de outros cursos da área da saúde como enfermagem, medicina e fisioterapia, encontraram resultados parecidos aos 
encontrados no presente estudo quanto à presença de excesso de peso: $17,9 \%$ e 18,4. Para Gasparetto \& Silva ${ }^{[3]}$, ao analisar o perfil antropométrico dos acadêmicos, também encontraram resultados semelhantes ao desta pesquisa, em que $64,2 \%$ dos estudantes apresentaram graus adequados de IMC.

De acordo com Anjos ${ }^{[14]}$, o IMC é benéfico para a classificação do estado nutricional em pesquisa com maiores amostras populacionais, contudo como avaliação separada não é um bom avaliador quando se trata da distribuição de gordura corporal. Portanto, medidas como a CC, RCQ e RCEst por exemplo, pode dar informações complementares quanto a presença ou não da obesidade e o risco pra complicações cardiovasculares.

O acúmulo de gordura abdominal foi determinado pela classificação da circunferência da cintura, identificando $8,3 \%$ da população estudada com risco elevado e $10 \%$ com risco muito elevado para doenças cardiovasculares e obesidade. Com observação de que a grande maioria dessa população, $81,7 \%$, estão em estado normal. Resultados semelhantes foram encontrados por Silva ${ }^{[2]}$ Observou-se que o percentual de estudantes que estavam com CC normal foi $84,29 \%$ e aqueles que estavam com CC em risco aumentado ou muito aumentado para doenças metabólicas na população total foi de $15,71 \%$. Já para os estudantes que apresentaram CC elevada e muito elevada de acordo com o sexo, observa-se que no público feminino o percentual foi maior $28,13 \%$, comparado com público masculino que apresentou 5,26\%. Quanto ao Percentual de Gordura Corporal (\%GC) total, 47,14\% estavam na faixa de risco à obesidade, sendo que a maioria era do sexo feminino $(81,25 \%)$, já o percentual para aqueles que estavam com \%GC desejável e limítrofe foi $52,85 \%$.

Em relação ao acúmulo de gordura abdominal, Gasparetto \& Silva ${ }^{[3]}$, também encontraram resultados semelhantes ao encontrado no nosso estudo, descrevendo que $19,8 \%$ dos universitários avaliados apresentavam risco elevado para doenças coronarianas. Foi analisado por esse mesmo inquérito antropométrico o perfil nutricional dos acadêmicos por período estudado, encontrado uma porcentagem significativa de cintura elevada no $7^{\circ}$ período e muito elevada no $1^{a}$ período.

Avaliamos esse mesmo inquérito por gênero da amostra estudada, foi observada que entre mulheres esse índice é predominante, sendo que apenas nas mulheres foram encontradas risco muito elevado da circunferência da cintura e apenas uma amostra foi detectada no sexo masculino para circunferência da cintura elevada. Sendo o estado nutricional preocupante no sexo feminino.

A média da CC de acordo com a mensuração realizada identificou uma média para esses estudantes de $77,50 \mathrm{~cm}$, indicando que não há risco para complicações metabólicas, conforme a classificação da OMS. Esse resultado foi semelhante ao encontrado por Gasparetto \& Silva ${ }^{[3]}$, onde os valores da CC para estudantes de nutrição foram de $78,42 \mathrm{~cm}$.

A relação entre as medidas de cintura e quadril utilizadas também para identificar gordura abdominal e risco de complicações metabólicas identificou apenas 08 ou seja 13,3\% de alunos com riscos para doenças cardiovasculares e predisposição para obesidade, sendo $52(86,7 \%)$ destes alunos sem risco para estas 
patologias. Considerando a Relação Cintura/Quadril (RCQ), o grupo feminino 13,3\% também apresentou-se predominantemente na classificação quanto ao risco para doenças cardiovasculares.

Dentre os dados levantados para compor as análises de risco cardiometabólico, a estatura do indivíduo, por ser relativamente imutável após idade adulta, tem servido como base para a composição de medidas mais aplicáveis às diversas populações. $A$ razão da medida de cintura pela estatura (RCE) é considerada útil para identificar sujeitos com alto risco metabólico e cardiovascular. Pitanga \& Lessa [15], demonstraram que a RCE é fortemente associada a diversos fatores de risco cardiovascular e identificam os pontos de corte mais próximos deste indicador antropométrico de obesidade para discriminar o risco coronariano, em diferentes populações. No entanto, são poucas pesquisas abrangendo a RCE em população universitária.

Neste estudo verificou-se que apenas as mulheres encontram em risco para doenças cardiovasculares e obesidade. Sendo apenas 13,3\% da amostra total, mas, tornando preocupante o estado nutricional das mulheres nesta pesquisa. Pode-se observar que todos as acadêmicas investigadas estão com risco diante da RCEst, corroborando com os dados a respeito do risco baseados na circunferência da cintura e Relação cintura - quadril. Estes resultados diferem dos resultados encontrados por Valadares et al. ${ }^{[16]}$, onde a prevalência de alto risco através da RCE observada na população de universitários foi elevada, principalmente por se tratar de um público jovem. Os resultados revelaram que mais da metade da amostra apresentou valores de RCE em alto risco, sendo que os homens tendem a apresentar valores mais acentuados que as mulheres, ou seja, o alto risco é maior entre os homens $(59,5 \%)$ em relação as mulheres (40,4\%). Semelhantes aos resultados encontrados nesta pesquisa, outro estudo que avaliou a RCE de adultos na cidade de Salvador/BA, encontrou maiores médias na população feminina ${ }^{[15]}$. Não foram encontrados outros estudos avaliando a RCE de população universitária em outras cidades do Brasil. Compete observar que a RCE tem sido apontada por algumas pesquisas como um marcador de risco mais efetivo que o IMC..

A avalição de consumo de alimentos é importante como indicador indireto do estado nutricional. Os inquéritos dietéticos são métodos utilizados para avaliação do consumo alimentar de indivíduos e populações em um determinado período de tempo estabelecido previamente. O QFA é usado na abordagem do individuo sobre seu consumo de determinado alimentos e bebidas, é um método qualitativo frequentemente utilizado na investigação sobre a relação entre composição da dieta e saúde, como na etiologia de doenças crônicas ${ }^{[17,7]}$.

O feijão e o arroz, há muito tempo são considerados itens básicos do padrão alimentar brasileiro. Para Marinho et al. ${ }^{[18]}$, também encontraram o consumo satisfatório de feijões entre adultos em outros Estudos realizados em diferentes municípios brasileiros. Diferentes dos nossos resultados, um estudo realizado por Santos et al. ${ }^{[19]}$, foi encontrado um índice de consumo diário de feijão apenas $28,33 \%$, sendo o maior índice de consumo semanal (52,5\%). O Guia Alimentar Brasileiro Brasil[20] indica uma porção de leguminosas por dia, que incluem vários tipos de feijão e outros alimentos como ervilhas, lentilhas e grão-de-bico. Todas as leguminosas são 
fontes de proteína, vitaminas do complexo B e minerais, como ferro, zinco e cálcio, e fibras e não devem ser excluídas da alimentação.

Entre os vegetais pesquisados os mais consumidos pelos acadêmicos diariamente são: cheiro verde, cebola, alho, pimenta de cheiro, tomate, pepino, couve e alface, sendo que esses alimentos foram os com maior percentual de consumo em relação as variáveis desse grupo. Outro estudo relatou resultados parecidos com o desta pesquisa onde Ramalho et al.[21], verificaram no seu estudo que a baixa prevalência de consumo regular de frutas e hortaliças de $14,8 \%$ foi evidenciada em acadêmicos da Universidade Federal do Acre, campus de Rio Branco. Ponderando estes estudos, nota-se o baixo consumo deste grupo de alimentos, o que traz apreensão, já que estes têm papel essencial na prevenção de algumas doenças, mas para isso é indispensável que seja consumido de forma variada e em quantidade mínima diariamente. Através desta recomendação, pode-se observar que a recomendação preconizada não foi alcançada pela amostra do nosso estudo, já que a maioria dos acadêmicos relataram consumir em pouca quantidade.

Dentre o grupo das oleaginosas, observou-se o pouco consumo diariamente e semanalmente dessa classe de alimentos. Os acadêmicos relatam o maior consumo por mês. No meio do grupo das Farinhas, féculas e massas os produtos mais consumidos são a farinha de mandioca e o macarrão.

No grupo de panificados o pão caseiro e pão francês são predominantes no consumo diário desses acadêmicos. Para Santos et al. ${ }^{[19]}$, foi encontrado que o consumo de pães $(41,66 \%)$ teve maior frequência de consumo semanal. Porém, para o consumo diário de 1-2x por dia os resultados também foram significantes $(35,83 \%)$. Souza [22], obteve achados semelhantes já que $42,2 \%$ de sua amostra relatou consumir pães todos os dias. A grande frequência no consumo de pães pelos estudantes foi confirmada por Gambardella et al. ${ }^{[23]}$, que o atribuíram à crescente substituição do jantar por lanches, mais práticos e saborosos.

O consumo de ovos entre os estudantes foi alto quando comparado com o consumo carnes bovinas, de peixes, vísceras, embutidos e aves. No estudo de Carvalho et al.,[24], comparou a frequência de consumo dos grupos alimentares entre estudantes do $1^{\circ}$ e do $4^{\circ}$ ano de nutrição, a maior frequência encontrada, em relação ao grupo de carnes e ovos, foi semanal, tanto nos acadêmicos do $1^{\circ}$ ano quanto do $4^{\circ}$ ano. No estudo realizado por Santos et al. ${ }^{[19]}$, verificou-se que os universitários consomem mais carne bovina do que peixes. Resultados diferentes encontrados nas nossas pesquisas.

O leite integral em pó está entre os laticínios, mas consumidos pelos estudantes diariamente, sendo ainda considerado um consumo baixo, pois, apenas $40 \%$ da amostra afirmou consumir leite. Resultado semelhante foi descrito no estudo feito por Leite $^{[25]}$, em que $(69,1 \%)$ dos estudantes também não consumiam leite. Já no estudo de Garcia et al. ${ }^{[26]}$, a maioria dos alunos portugueses (56\%) afirmaram consumi-lo todos os dias e apenas $10,4 \%$ afirmaram não o consumir. No estudo realizado por Santos et al. ${ }^{[19]}$. Em relação ao consumo de leites e derivados, tivemos uma média de 1,74 porções/dia, sendo o seu consumo inadequado de acordo com as recomendações da pirâmide alimentar utilizada nesse estudo que recomendam 3 
porções diárias. Marcondelli et al. ${ }^{[2]}$, também encontraram um percentual baixo de adequação para consumo de leite e derivados entre universitários. Maciel et al. ${ }^{[28]}$, nos seus estudos com universitários encontraram resultados inadequados (23\%) no consumo de leite e derivados. Em estudo de Santos et al. ${ }^{[19]}$, observou-se que os produtos lácteos eram raramente consumidos por $50 \%$ a $70 \%$ dos entrevistados. Sendo estes resultados parecido com o trabalho pesquisado.

Verificou-se que os acadêmicos consomem com frequência alimentos industrializados devido a praticidade de serem preparados. O problema que advém dessa ação é o efeito colateral de tantos produtos adicionados nos alimentos o corpo fica exaurido (mesmo sem ter feito exercício algum) e fica difícil se concentrar, se inspirar e resolver problemas. Os alimentos processados aumentam a letargia e abaixam a motivação, além de estarem ligados a aumento de casos de depressão e agressividade.

O registro alimentar 24 horas é um método quantitativo cujo o principal objetivo é conhecer o conteúdo de calorias, macro e micronutrientes ingeridos. Analisando o registro alimentar 24 horas podemos mostrar que o consumo de macronutrientes demonstrou uma prevalência normal no consumo de proteínas e baixa no consumo de lipídeos $(48,3 \%)$, assim como no consumo de carboidratos, quase $(68,3 \%)$ da amostra.

Diferente do estudo realizado por Souza [12], em relação ao consumo de macronutrientes, ambos os grupos apresentaram uma prevalência de consumo alto de proteínas e de lipídeos (>50\%) e baixo em carboidratos, quase $50 \%$ da amostra. Sabe-se que o consumo de carboidratos definido como ideal está entre 50 a $60 \%$ do total de calorias diárias ${ }^{[29]}$.

Os carboidratos são um grupo diverso de substâncias com propriedade fisiológicas, físicas e químicas características. Essas propriedades Como são primordialmente substratos para o metabolismos energético, podem afetar a saciedade, glicemia, a insulinemia e o metabolismo lipídico. Essas propriedades têm implicações sobre a saúde em geral e contribuem para o controle do peso corporal, do envelhecimento e etc., [30]. Há uma grande prevalência $(68,3 \%)$ de acadêmicos estudados que não ingerem a quantidade de carboidratos adequados, $18,4 \%$ consomem acima do recomendando e apenas $13,3 \%$ dos acadêmicos consomem a quantidade recomendada, sendo o maior consumo de carboidratos descritos para os alunos do $9^{a}$ período. Estudos realizados por Souza ${ }^{[18]}$, demostram resultados semelhantes, com a prevalência de consumo baixo de carboidratos $(<50 \%$ do total de calorias diárias. Quanto ao consumo baixo de carboidratos, sugere-se que isso reflete de uma compensação de baixo consumo de lipídeos. Sabe-se que os carboidratos são uma importante fonte de energia para o corpo e que na sua ausência, outros nutrientes desempenharão seu papel, prejudicando o crescimento e desenvolvimento dos indivíduos ${ }^{[29]}$.

A recomendação do consumo de proteínas é de 10 a 15\% das calorias diárias. As proteínas são de extrema importância para o nosso organismo por sua função construtora e reparadora. Elas também participam da formação de hormônios, enzimas e anticorpos, Por outro lado, sua falta pode causar inúmeros problemas como 
fadiga, queda de cabelo e até baixa imunidade ${ }^{[30]}$. A prevalência geral de consumo alto de proteínas foi de $26,7 \%$, apenas $25 \%$ da amostra consumia valores abaixos de $15 \%$ das calorias diárias recomendadas, e uma prevalência de $48,3 \%$ das recomendações diárias desejadas. Diferente do estudo realizado por Souza ${ }^{[12]}$, que observou um consumo alto de proteínas ( $>15 \%$ do total das calorias diárias) de $62,1 \%$ e uma prevalência de consumo baixo de proteínas ( $<10 \%$ das calorias diárias) de $8,6 \%$ nos universitários.

Os lipídios são moléculas orgânicas formadas a partir de ácidos graxos e álcool que desempenham importantes funções no organismo dos seres vivos, funcionando, entre outras atividades, como eficiente reserva energética ${ }^{[30]}$. Analisano o consumo de lipídeos, foi encontrado que $31,7 \%$ dos indivíduos estão acima dos valores recomendados (>30\% do consumo total de calorias), $48,3 \%$ estão abaixo desse consumo diário, e apenas $20 \%$ dos alunos apresentam o consumo recomendado. Resultados esses que diferem do encontrado por Souza ${ }^{[12]}$, onde a prevalência geral de consumo alto de lipídeos foi de $55,2 \%$ e a prevalência de consumo baixo foi de $19 \%$ na amostra total, sendo este valor similar em ambos os semestres avaliados.

As fibras são importantes na alimentação porque aceleram a passagem dos produtos residuais do organismo, absorvem substâncias perigosas (toxinas) e mantém o tubo digestivo saudável[ ${ }^{[30]}$. O consumo de fibras inadequado ressaltou em $78,3 \%$ da amostra estudada que consomem menos que 20-30 gramas de fibras por dia, sendo que apenas o $9^{\circ}$ período chega mais próximo do consumo adequado de fibras. Acredita-se que os conhecimentos adquiridos pelos alunos do $9^{\circ}$ podem modificar os próprios hábitos alimentares e seu estado nutricional em relação ao demais períodos.

Esse foi o primeiro relato acerca do perfil alimentar dos estudantes de nutrição da Universidade Federal do Amazonas e os resultados reportados animam pesquisas futuras que possam considerar uma período mais abrangente de análise.

\section{CONCLUSÃO}

A alimentação dos acadêmicos de nutrição foram consideradas inadequadas, mesmo sendo encontrado uma grande quantidade de eutróficos e sem risco para obesidade e doenças cardiovasculares. Não foi encontrado alterações entre os mesmos no que se diz respeito a importância aos hábitos alimentares. Detectou-se uma baixa ingestão dos grupos alimentares considerados essenciais, como as frutas, legumes e verduras, leguminosas, carnes, leite e seus derivados.

Ao avaliar o registro alimentar 24 horas foi evidenciado o baixo consumo de macronutrientes como o carboidratos e fibra alimentar segundo as recomendações nutricionais para adultos saudáveis. 
De modo geral os acadêmicos de nutrição precisam de uma atenção maior, pois, assim como foi evidenciado, nem sempre o que é aprendido na teoria é realizado na prática.

Análises nesta área são indispensáveis para elaboração de estratégias para a promoção da saúde e para a prevenção de doenças no meio universitário. $O$ conhecimento sobre a importância dos cuidados nutricionais é essencial, e tem uma repercussão para a vida como um todo desses indivíduos. Seria estimável que todos os cursos de graduação na área da saúde contemplassem o tema da alimentação saudável em sua formação, método que ajudaria no estabelecimento de hábitos mais saudáveis e promoção da saúde.

\section{REFERÊNCIA}

1. Alves HJ, Boog MAF. Comportamento alimentar em moradia estudantil: um espaço para a promoção da saúde. Rev Saúde Púb. 2007; 41(2): 197-204.

2. Silva LR, Fonseca MCP, Lira CRN, Assis BS, Alonso CMP. Estado Nutricional e Estilo de Vida de Estudantes de um Restaurante Universitário da Cidade de Salvador - BA, Brasil. Rev Inter de Lingua Port. 2018; 33: 131 - 146.

3. Gasparetto RM., Silva RCC. Perfil antropométrico dos Universitários dos cursos de nutrição, enfermagem, fisioterapia e educação física do centro universitário LA SALLE, CANOAS/RS. Rev da Asso Bras de Nutr. 2014; 4(1): 29-33.

4. World Health Organization - WHO. Physical Status: The Use and Interpretation of Anthropometry. Geneva: WHO; 1995.

5. Matias CT, Fiore EG. Mudanças no comportamento alimentar de estudantes do curso de nutrição em uma instituição particular de ensino superior. Nutrire: ver. Soc. Bras. Alim. Nutr. J. Brazilian Soc. Food Nutr. Ago/2010; 35(2):53-66.

6. Oliveira JS, Santos DO, Rodrigues SJM, Oliveira CC, Souza ALC. Avaliação do perfil sociodemográfico, nutricional e alimentar de estudantes de nutrição de uma universidade pública em Lagarto-SE. Rasbran. Jul-Dez. 2017; 8(2):37-42.

7. Slater B, Philippi ST, Marchioni DML, Fisberg RM. Validação de Questionários de Frequência Alimentar - QFA: Considerações Metodológicas. Rev Bras Epidemiol. 2003; 6(3): 200-209.

8. Linhares ASL. Consumo alimentar fora de casa, seus fatores determinantes e associação ao padrão alimentar mediterrâneo. Trabalho de investigação. Porto, 2012. 
9. Feitosa EPS, Dantas CAO, Andradewartha ERS, Marcellini PS, Mendesnetto RS. Hábitos alimentares de estudantes de uma universidade pública no nordeste, Brasil. Alim. Nutr. abr./jun. 2010; 21(2): 225-230.

10. Ramos SA. Avaliação do estado nutricional de universitários. [Dissertação]. Belo Horizonte: Faculdade de farmácia da UFMG. 2005.

11. Munhoz PM, Oliveira J, Anjos JC, Gonçalves RD, Lopes JF, Celemi LG. Perfil nutricional e hábitos alimentares de universitários do curso de bnutrição. Rev Saúde UniToledo. set./nov. 2017. 01(02): 68-85.

12. Souza DP. Avaliação do estado nutricional e consumo alimentar de acadêmicos do curso de nutrição da Universidade Federal de Pelotas - Rio Grande. Pelotas: HCPA, 2012.

13. Simão CB, Nahas MV, Oliveira ESA. Atividade física habitual, hábitos alimentares e prevalência de sobrepeso e obesidade em universitários da Universidade do Planalto Catarinense - UNIPLAC. Rev Bras Ativ Fís Saúde. 2008.

14. Anjos LA. Índice de massa corporal (massa corporal.estatura-2) como indicador do estado nutricional de adultos: revisão da literatura. Rev Saúde Pública [periódico na internet]. 1992 Dez [citado 2014 Maio 12]; 26(6): 431-36. Piasetzki CTR; Bracht CM; Busnello MB; Winkelmann ER; Boff ETO; Franz LBB. Consumo Alimentar e Prática de Atividade Física em Estudantes Universitários. Revista Biomotriz, 2012; 6(1).

15. Pitanga FJG, Lessa I. Razão cintura-estatura como discriminador do risco coronariano de adultos. Rev da Asso Méd Bras. 2006; 52(3):157-161.

16. Valadares AS, Santos CA, Rovella C. et al., Relação cintura-estatura de universitários de uma faculdade particular do município de praia grande, sp. Rev Acadêmica Institucional. Ano viii - nº xviii- jul/ 2015.

17. Marchioni DML, Slater B, Fisberg RM. Aplicação das Dietary Reference Intakes na avaliação da ingestão de nutrientes para indivíduos. Rev de Nutr. 2004; 17(2):207-216.

18. Marinho MCS, Hamann EM, Lima ACF. Práticas e mudanças no comportamento alimentar na população de Brasília, Distrito Federal, Brasil. Rev Bras Saú Mater Infa. jul./set. 2007; 7(3): 251-261.

19. Santos AG, Reis CC, Chaud DM et al,. Qualidade de vida e alimentação de estudantes universitários que moram na região central de são paulo sem a presença dos pais ou responsáveis. Rev. Simbio-Logias. Dez/2014; 7(10).

20. Brasil. Ministério da Saúde. Secretaria de Atenção à Saúde. Coordenação-Geral da Política de Alimentação e Nutrição. Guia alimentar para a população brasileira: promovendo a alimentação saudável. Brasília: Ministério da Saúde, 2014. 
21. Ramalho AA, Dalamaria T, Souza OF. Consumo regular de frutas e hortaliças por estudantes universitários em Rio Branco, Acre, Brasil: prevalência e fatores associados. Caderno de Saúde Pública, v. 28, n. 7, p. 1405-1413, 2012.

22. Souza MM. Estado nutricional, prática de atividade física e hábitos alimentares de acadêmicos de enfermagem [monografia]. Paraíba: Universidade Estadual da Paraíba; 2011.

23. Gambardella, AMD, Frutuoso MFP, Franchi C. Prática alimentar de adolescentes. Rev. de Nutr. 1999; 12: 55-63.

24. Carvalho CS, Santos AA, Vigato TC, Paese MCS. Frequência de consumo de grupos alimentares por acadêmicas do $1^{\circ}$ e $4^{\circ}$ ano nos cursos de nutrição e pedagogia das Faculdades Unidas do Vale do Araguaia no município de Barra do Garças - MT. Rev da Univar. 2013; 2(10):78-82.

25. Leite ACB. Qualidade de vida e condições de saúde de acadêmicos de nutrição. Rev Esp para a Saúde.2011;13(1):82-90.

26. Garcia D, Nabo G, Aleixo V, Correia V, Ribeiro I, Costa MC. Physical Activity and Dietary Habits in a University Population. Biomed Biopharm Res. 2013; 9(2): 147-158.

27. Marcondelli PC, Costa THM, Schmitz BAS. Nível de atividade física e hábitos alimentares de universitários do $3^{\circ}$ ao $5^{\circ}$ semestres da área da saúde. Rev de Nutr. 2008; 21(1):39-47.

28. Maciel ES, Sonati JG, Modeneze DM, Vasconcelos JS, Vilarta R. Consumo alimentar, estado nutricional e nível de atividade física em comunidade universitária brasileira. Rev de Nutr. 2012; 25(6):707-718.

29. Mahan LK, Escoff-Stump S. Krause: Alimentos, Nutrição e Dietoterapia. $12^{\circ}$ ed. São Paulo: Elsevier, 2010. 1358p.

30. Cozzolino SMF, Cominetti C. Bases bioquímicas e fisiológicas da nutrição: nas diferentes fases da vida, na saúde e na doença. Barueri, SP: Manole,2013.

Artigo recebido em: $27 / 04 / 2020$

Artigo aprovado em: 24/09/2020

Artigo publicado em: 26/10/2020 